\title{
YAGM: a web tool for mining associated genes in yeast based on diverse biological associations
}

\author{
Wei-Sheng Wu', Chung-Ching Wang, Meng-Jhun Jhou, Yu-Cheng Wang \\ From Joint 26th Genome Informatics Workshop and Asia Pacific Bioinformatics Network (APBioNet) 14th \\ International Conference on Bioinformatics (GIW/InCoB2015) \\ Tokyo, Japan. 9-11 September 2015
}

\begin{abstract}
Background: Investigating association between genes can be used in understanding the relations of genes in biological processes. STRING and GeneMANIA are two well-known web tools which can provide a list of associated genes of a query gene based on diverse biological associations such as co-expression, co-localization, co-citation and so on. However, the transcriptional regulation association and mutant phenotype association have not been used in these two web tools. Since the comprehensive transcription factor (TF)-gene binding data, TF-gene regulation data and mutant phenotype data are available in yeast, we developed a web tool called YAGM (Yeast Associated Genes Miner) which constructed the transcriptional regulation association, mutant phenotype association and five commonly used biological associations to mine a list of associated genes of a query yeast gene.

Description: In YAGM, we collected seven kinds of datasets including TF-gene binding (TFB) data, TF-gene regulation (TFR) data, mutant phenotype (MP) data, functional annotation (FA) data, physical interaction (PI) data, genetic interaction (GI) data, and literature evidence (LE) data. Then by using the hypergeometric test to calculate the association scores of all gene pairs in yeast, we constructed seven biological associations including two transcriptional regulation associations (TFB association and TFR association), MP association, FA association, PI association, Gl association, and LE association. Moreover, the expression profile association from SPELL database was also included in YAGM. When using YAGM, users can input a query gene and choose any possible subsets of the eight biological associations, then a list of associated genes of the query gene will be returned based on the chosen biological associations.

Conclusions: In this study, we presented the YAGM which provides eight biological associations for mining associated genes of a query gene in yeast. Among the eight biological associations constructed in YAGM, three (TFB association, TFR association, and MP association) are novel ones. By comparing the query results of two wellknown web tools (STRING and GeneMANIA), we found that YAGM can find out distinct associated genes of a query gene. That is, YAGM can provide alternative candidates of associated genes for biologists to do further experimental investigation. We believe that YAGM will be a useful web tool for yeast biologists. YAGM is available online at http://cosbi3.ee.ncku.edu.tw/yagm/.
\end{abstract}

\section{Background}

Exploring the association between genes is a crucial issue in the biology study. It helps biologists to discover the relationship of genes. For example, functional annotation association can be used to predict unknown functions of genes [1], expression profile association can be

\footnotetext{
* Correspondence: wessonwu@mail.ncku.edu.tw Tainan, Taiwan

Department of Electrical Engineering, National Cheng Kung University, tional regulation association can be used to predict coregulated genes [3]. Therefore, it would be helpful if there are web tools which can provide a list of associated genes of a query gene based on diverse biological associations.

STRING [4] and GeneMANIA [5] are two well-known web tools which can provide this kind of services. These two tools return a list of associated genes of a query 
gene based on diverse biological associations derived from neighbourhood, gene fusion, co-occurrence, coexpression, co-localization, co-citation, co-inheritance, genetic interaction, physical interaction, shared protein domains and so on. Although many kinds of biological associations have been used in these two web tools, the transcriptional regulation association and the mutant phenotype association have not been considered yet. Therefore, given a query gene, these two tools cannot provide a list of genes which have similar transcriptional regulatory mechanisms or similar mutant phenotypes to the query gene. Since the comprehensive transcription factor (TF)-gene binding data, TF-gene regulation data and mutant phenotype data are available in yeast, this gives us an opportunity to construct the transcriptional regulation association and the mutant phenotype association in yeast. Moreover, we have many experiences in developing databases and web tools [6-13].

So here we are able to present a web tool called YAGM (Yeast Associated Genes Miner) which constructs eight biological associations to mine a list of associated genes of a query yeast gene. These biological associations include three novel ones (TF binding association, TF regulation association and mutant phenotype association) and five commonly used ones (functional annotation association, physical interaction association, genetic interaction association, literature evidence association, and expression profile association). Depending on the selected biological associations, YAGM can provide a list of genes which have similar bound TFs, similar regulatory TFs, similar mutant phenotypes, similar functions, similar physical interactions, similar genetic interactions, similar literature evidences, or similar expression profiles to the query gene. Moreover, YAGM has a user-friendly search interface and the search results are visualized as network graphs and tables.

\section{Construction and Contents Construction of YAGM}

In YAGM, we collected seven kinds of datasets including TF-gene binding (TFB) data, TF-gene regulation (TFR) data, mutant phenotype (MP) data, functional annotation (FA) data, physical interaction (PI) data, genetic interaction (GI) data, and literature evidence (LE) data. Then by using the hypergeometric test to calculate the association scores of all gene pairs in yeast, we constructed seven biological associations including TFB association, TFR association, MP association, FA association, PI association, GI association, and LE association. Moreover, the expression profile (EP) association from SPELL database [2] was also included in YAGM. When using YAGM, users can input a query gene and select any possible subsets of the eight biological associations, then a list of associated genes of the query gene will be returned based on the chosen biological associations (see Figure 1).

\section{Data collection}

Seven kinds of genome-wide datasets were gathered to construct the seven biological associations. First, 41,013 TF-gene binding pairs were retrieved from the YEASTRACT database [14]. Each TF-gene binding pair has experimental evidence (from band-shift, foot-printing or ChIP assay) showing that the TF binds to the promoter of the gene. Second, 168,900 TF-gene regulation pairs were retrieved from the YEASTRACT database. Each TF-gene regulation pair has experimental evidence (from detailed gene by gene analysis or genome-wide expression analysis) showing that the TF perturbation (knockout or over-expression) causes a significant change in the expression of the gene. Third, 605 mutant phenotypes with 10 diverse mutant types were retrieved from SGD database [15]. Fourth, 1,362 yeast functional annotations with 28 main functional categories were retrieved from MIPS database [16]. Fifth, 120,579 physical interactions were retrieved form BioGRID database [17]. Sixth, 190,196 genetic interactions were also retrieved from BioGRID database. Seventh, 70,674 publications associated with genes of interest were downloaded from SGD database.

\section{Calculation of association scores}

A previous study [18] investigated the performance of different association measures (Jaccard index, cosine index, Pearson correlation index and hypergeometric index) in calculating the statistical significance of the overlap of two sets. They found that hypergeometric index performed better than the other indices. Therefore, for each of the seven biological associations, we adopted hypergeometric index, shown in Equation (1), to calculate the association score between the query gene $a$ and another gene $b$ :

$$
H_{i}(a, b)=-\log \left(\sum_{x \geq k}^{\min (m, n)} \frac{\left(\begin{array}{c}
m \\
x
\end{array}\right)\left(\begin{array}{c}
N-m \\
n-x
\end{array}\right)}{\left(\begin{array}{c}
N \\
n
\end{array}\right)}\right)
$$

where $i=$ TFB, TFR, MP, FA, PI, GI and LE. The definition of each parameter in Equation (1) is given in Table 1. For example, PI association between genes $a$ and $b$ measures the significance of the overlap of two sets. The first one is the set of proteins which have physical interactions with the protein product of gene $a$ and the second one is the set of proteins which have physical interactions with the protein product of gene $b$.

In addition, the expression profile (EP) association $\mathrm{H}_{E P}$ $(a, b)$ between the query gene $a$ and another gene $b$ was 


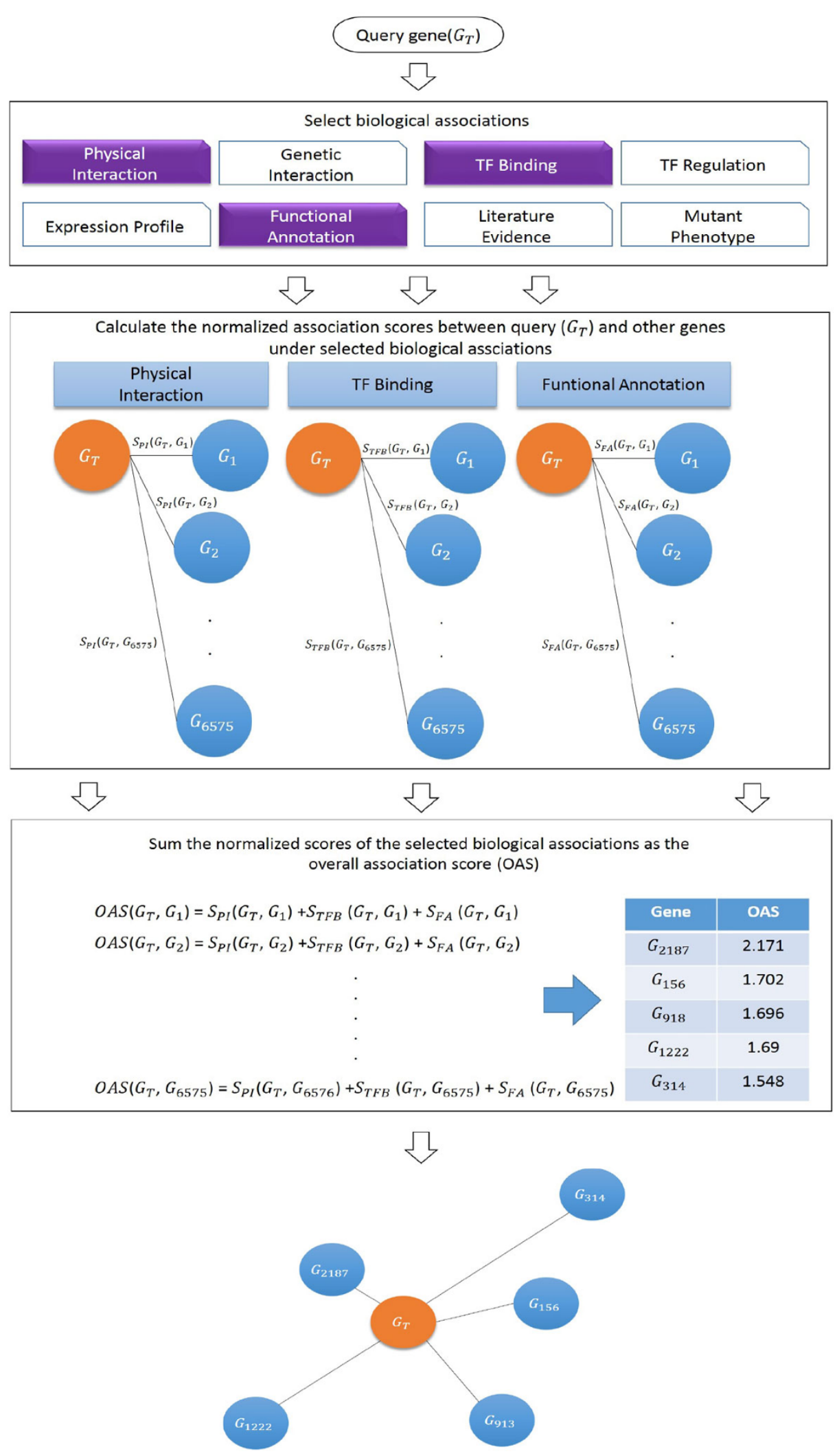

Figure 1 Construction of YAGM. 
Table 1. Parameters of the Hypergeometic test for the seven constructed biological annotations

\begin{tabular}{|c|c|}
\hline Biological Association & Parameters of the Hypergeometric Test \\
\hline TF Regulation/TF Binding & $\begin{array}{l}\mathrm{N}: \text { \# of TFs collected from YEASTRACT } \\
\mathrm{n} \text { : \# of TFs that regulate/bind to gene } a \\
\mathrm{~m}: \text { \# of TFs that regulate/bind to gene } b \\
\mathrm{k}: \text { \# of TFs that regulate/bind to both gene } a \text { and gene } b\end{array}$ \\
\hline Mutant Phenotype/Literature Evidence & $\begin{array}{l}\mathrm{N}: \text { \# of mutant phenotypes/literature evidences collected from SGD } \\
\mathrm{n} \text { : \# of mutant phenotypes/literature evidences of gene } a \\
\mathrm{~m}: \text { \# of mutant phenotypes/literature evidences of gene } b \\
\mathrm{k}: \text { \# of mutant phenotypes/literature evidences of both gene } a \text { and gene } b\end{array}$ \\
\hline Functional Annotation & $\begin{array}{l}\mathrm{N}: \text { \# of functional annotations collected from MIPS } \\
\mathrm{n} \text { : \# of functional annotations of gene } a \\
\mathrm{~m} \text { : \# of functional annotations of gene } b \\
\mathrm{k} \text { : \# of functional annotations of both gene } a \text { and gene } b\end{array}$ \\
\hline Physical Interaction/Genetic Interaction & $\begin{array}{l}\mathrm{N}: \text { \# of genes in the yeast genome } \\
\mathrm{n} \text { : \# of genes that have physical/genetic interactions with gene } a \\
\mathrm{~m}: \text { \# of genes that have physical/genetic interactions with gene } b \\
\mathrm{k} \text { : \# of genes that have physical/genetic interactions with both gene } a \text { and gene } b\end{array}$ \\
\hline
\end{tabular}

retrieved directly from SPELL database [2]. Subsequently, we used Equation (2) to normalize $\mathrm{H}_{i}(a, b)$ into the range $[0,1]$ as follows:

$$
S_{i}(a, b)=\frac{H_{i}(a, b)-\min _{b} H_{i}(a, b)}{\max _{b} H_{i}(a, b)-\min _{b} H_{i}(a, b)}
$$

Finally, we summed the normalized scores of the chosen biological associations as the overall association score $(O A S)$ between the query gene $a$ and another gene $b$ shown in Equation (3):

$$
\operatorname{OAS}(a, b)=\sum \begin{gathered}
t \in \text { chosen } \\
\text { biological } \\
\text { association }
\end{gathered} S_{i}(a, b)
$$

\section{Implementation of the web service of YAGM}

The web interface of YAGM is constructed using the PHP language with the CodeIgniter MVC framework. Basic information of yeast genes and scores of eight biological associations for each gene pairs are deposited in MySQL. The table showing the list of associated genes of the query gene is produced by the JQuery. The network graph containing the query gene and all its associated genes is generated by Cytoscape [19].

\section{Utility and discussion Web interface}

YAGM provides four web pages (the query page, the search result page, the detail page and the reference page) to present the information of a list of associated genes of a query gene based on the selected biological associations. In the query page (Figure 2), users can input a yeast gene name, set the number of associated genes being reported, and select the biological associations being used.

After submission, users will get a search result page, which can be divided into three parts. The first part
(Figure 3a) contains the basic information (name, chromosome location, description, sequence and MIPS functional catalogue) of the query gene. The second part (Figure 3b) contains two network graphs connecting the query gene with all its associated genes. The first network graph is called the confidence view. The edge between the query gene and its associated gene in the network reflects the overall association score (OAS). The higher the OAS, the wider and shorter the edge. The second network graph is called the evidence view. The edge between the query gene and its associated gene in the network indicates that this gene pair has the evidence of a specific biological association. This means that the association score of this gene pair under that biological association is higher than the 95th percentile of the association scores of all gene pairs in the yeast genome. The third part (Figure 3c) is a table listing the associated genes. In the table, the information of each associated gene contains the evidences of specific biological associations, the OAS, and a link of "Detail".

When clicking the link of "Detail", users will be directed to the detail page. The detail page (Figure 4a) reveals how the score of each chosen biological association between the query gene and its associated gene is calculated. For example, when calculating the TFB association score between the query gene FKS1 and its associated gene GSC2 using Equation (1), we need to know the list of TFs which bind to FKS1, the list of TFs which bind to GSC2, and the list of TFs which bind to both FKS1 and GSC2. The original resources which provide these three lists of TFs are shown in the reference page (Figure 4b).

\section{Case study}

FKS1 is a protein involved in cell wall synthesis and maintenance [15]. Here we input FKS1 as a query gene and use all eight biological associations. Then the top five associated genes returned by YAGM is shown in Figure 3c. It can be seen that all these five associated 


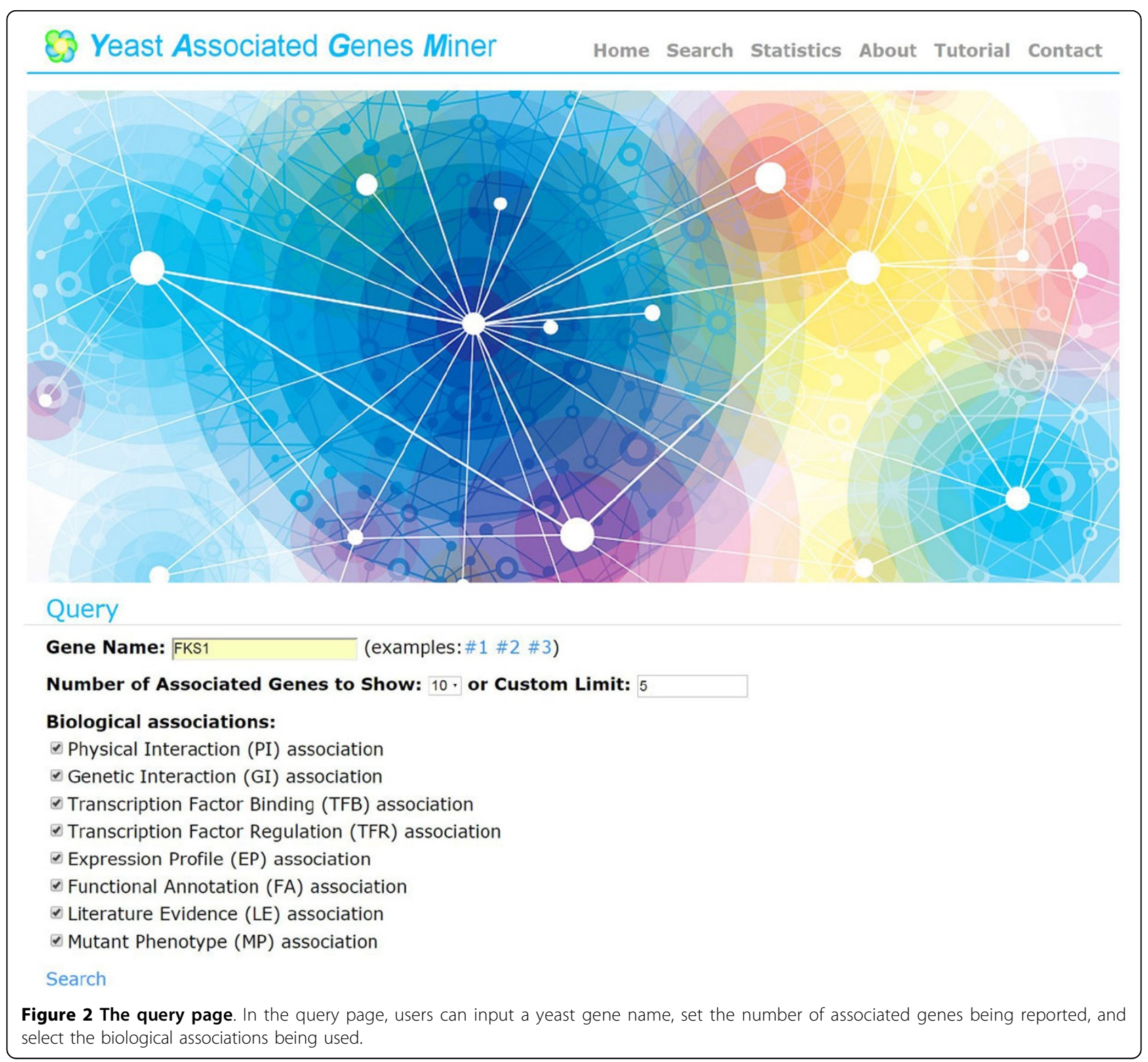

genes have at least six evidences of biological associations, suggesting that they are associated with the query gene in terms of diverse biological associations. We then check the biological plausibility of these five associated gene by using the gene description content in SGD database [15]. Four (GSC2, GAS1, SMI1, CCW12) of the five predicted associated genes are known to be involved in cell wall assembly or synthesis just like the query gene FKS1, suggesting that YAGM can predict biologically plausible associated genes of a query gene.

\section{Investigation of the relationships between different biological associations}

In order to see how well the different biological associations correlate, for each query gene, we compared the two lists of top 50 associated genes using two different biological associations, respectively. The same process was done for all 6576 possible query genes. Then the average overlap and standard error could be computed (see Additional file 1). We found that the two lists of top 50 associated genes using two different biological associations have low overlap most of the time, indicating different biological associations are usually lowly correlated. The only exception is the TFB-TFR pair. These two biological associations are highly correlated.

Moreover, in order to know which biological associations are more related to the OAS than the others, for each query gene, we compared the two lists of top 50 associated genes using all eight biological associations together and only one biological association, respectively. 
(a)

\begin{tabular}{|c|c|}
\hline ORF Name & YLR342W \\
\hline Gene Name & FKS1 \\
\hline Alias & CND1,CWH53,ETG1 \\
\hline $\begin{array}{l}\text { Chromosome } \\
\text { Location }\end{array}$ & ChrXII: 809997 to 815627 \\
\hline Description & $\begin{array}{l}\text { Catalytic subunit of } 1,3 \text {-beta-D-glucan synthase; functionally redundant with alternate catalytic } \\
\text { subunit Gsc2p; binds to regulatory subunit Rho1p; involved in cell wall synthesis and maintenance; } \\
\text { localizes to sites of cell wall remodeling; FKS1 has a paralog, GSC2, that arose from the whole } \\
\text { genome duplication }\end{array}$ \\
\hline Sequence & Sequence \\
\hline $\begin{array}{l}\text { MIPS } \\
\text { Functional } \\
\text { Catalogue }\end{array}$ & $\begin{array}{l}+01 \text { : Metabolism } \\
+02 \text { :Energy } \\
+42 \text { : Biogenesis of cellular components }\end{array}$ \\
\hline
\end{tabular}

(b.1)

Network
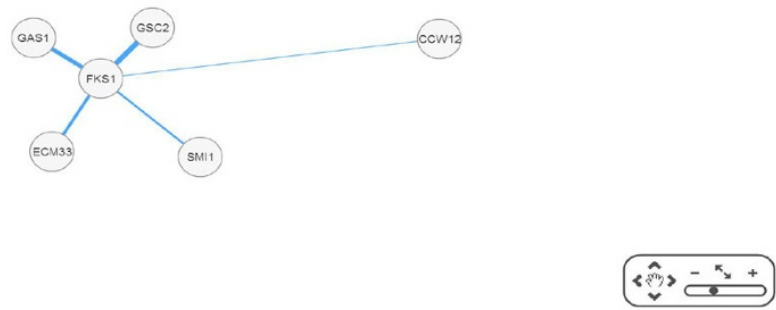

(b.2)

Network
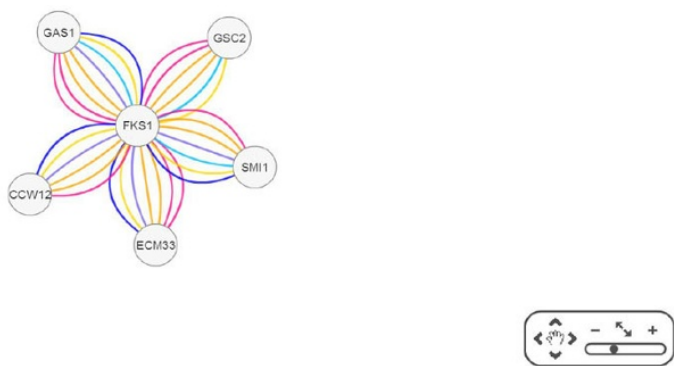

Note:

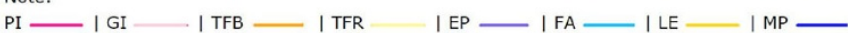

(c) Associated genes of the query gene

\begin{tabular}{|c|c|c|c|c|c|c|c|c|c|c|c|}
\hline \multicolumn{6}{|c|}{ Show $50 \cdot$ entries } & \multicolumn{6}{|c|}{ Search: } \\
\hline ORF name & Gene name & PI & GI & TFB & TFR & EP & FA & LE & MP & OAS & Detail \\
\hline YGR032W & GSC2 & 0 & 0 & $\circ$ & 0 & & 0 & 0 & & 4.869 & Detail \\
\hline YMR307W & GAS1 & o & o & o & o & o & o & 0 & o & 4.587 & Detail \\
\hline YBR078W & ECM33 & 0 & $\circ$ & $\circ$ & 0 & 0 & & 0 & 0 & 4.117 & Detail \\
\hline YGR229C & SMI1 & & 0 & 0 & 0 & 0 & o & o & o & 4.033 & Detail \\
\hline YLR110C & CCW12 & & o & o & 0 & 0 & & 0 & o & 3.965 & Detail \\
\hline
\end{tabular}

Figure 3 The search result page. The search result page can be divided into three parts. (a) The first part contains the basic information (name, chromosome location, description, sequence, and MIPS functional catalogue) of the query gene. (b) The second part contains two network graphs connecting the query gene with all its associated genes. The first network graph is called the confidence view. The edge between the query gene and its associated gene in the network reflects the overall association score (OAS). The higher the OAS, the wider and shorter the edge. The second network graph is called the evidence view. The edge between the query gene and its associated gene in the network indicates that this gene pair has the evidence of a specific biological association. This means that the association score of this gene pair under that biological association is higher than the 95th percentile of the association scores of all gene pairs in the yeast genome. (c) The third part is a table listing the associated genes. In the table, the information of each associated gene contains the evidences of specific biological associations, the OAS, and a link of "Detail". 
(a)

\section{TF Binding}

Feature definition: The TFs that bind to the gene of interest.

Data source: Yeastract

Score: Hypergeometric Function

$$
S(a, b)=-\log \left(\sum_{x \geq k}^{\min (m, n)} \frac{\left(\begin{array}{l}
m \\
x
\end{array}\right)\left(\begin{array}{c}
N-m \\
n-x
\end{array}\right)}{\left(\begin{array}{l}
N \\
n
\end{array}\right)}\right)
$$

- N: The population of interaction.

- $\mathrm{n}$ : The quantity of interaction which associates with target gene.

- $\mathrm{m}$ : The quantity of interaction which associates with compared gene.

- k: The quantity of interaction which associates with target gene and compared gene.

And then normalized scores by scaling the range of scores are between 0 and 1 .

The quantity of transcription factor:

- YLR342W(FKS1): 23

- YGR032W(GSC2): 13

- Intersection: 10

\begin{tabular}{|c|c|c|}
$\begin{array}{c}\text { Show } 10 \cdot \text { entries } \\
\text { TF name }\end{array}$ & $\begin{array}{c}\text { Search: } \\
\text { YLR342W(FKS1) }\end{array}$ & 0 \\
\hline YRM1 & 1 & 0 \\
\hline YAP6 & 1 & 1 \\
\hline TEC1 & 2 & 0 \\
\hline SWI6 & 2 & 1 \\
\hline SWI4 & 3 & 0 \\
\hline SUM1 & 1 & 2 \\
\hline STE12 & 3 & 1 \\
\hline SPT23 & 1 & 0 \\
\hline SOK2 & 2 & 0 \\
\hline SKO1 & 2 & 4 Previous Next
\end{tabular}

(b)

Reference

Show $10 \cdot$ entries

\begin{tabular}{|c|c|c|c|c|c|c|}
\hline \multicolumn{4}{|l|}{ 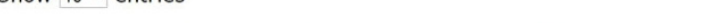 } & \\
\hline Gene & TF & Type & Author & $\begin{array}{l}\text { Association } \\
\text { Type }\end{array}$ & $\begin{array}{l}\text { Environmental } \\
\text { Condition }\end{array}$ & Pubmed \\
\hline YLR342W & SWI6 & $\begin{array}{l}\text { Binding:ChIP- } \\
\text { on-chip }\end{array}$ & $\begin{array}{l}\text { Simon I et al., Cell, } \\
2001 ; 6(6): 697-708\end{array}$ & $\begin{array}{c}\text { Not } \\
\text { applicable }\end{array}$ & $\begin{array}{l}\text { YPD medium; } \\
\text { mid-log phase }\end{array}$ & link \\
\hline YLR342W & SWI6 & $\begin{array}{l}\text { Binding:ChIP- } \\
\text { on-chip }\end{array}$ & $\begin{array}{l}\text { Workman CT et al., Science, } \\
2006 ; 312(5776): 1054-59\end{array}$ & NA & NA & link \\
\hline
\end{tabular}

Figure 4 The detail page and the reference page. (a) The detail page reveals how the score of each chosen biological association between the query gene and its associated gene is calculated. For example, when calculating the TFB association score between the query gene FKS1 and its associated gene GSC2, we need to know the list of TFs which bind to FKS1 and the list of TFs which bind to GSC2. Both lists of TFs are shown in the detail page. (b) The reference page provides the original resource of the data used for calculating the score of a biological association. 
The same process was done for all 6576 possible query genes. Then the average overlap and standard error could be computed (see Additional file 1). We found that the list of top 50 associated genes using all eight biological associations together have greater average overlap (14 out of 50) with the lists using only TFB association, only TFR association or only LE association than the lists using the other biological associations. This means that TFB association, TFR association and LE association are more informative than the other associations.

\section{Comparison with related databases}

STRING [4] and GeneMANIA [5] are two well-known web tools which can output a list of associated genes of a query gene based on diverse biological associations. Since these two tools provide the same service as our YAGM does, it is informative to do some comparisons. First, we compare the biological associations used in these three tools. As shown in Table 2 four biological associations (physical interaction, genetic interaction, co-expression and co-citation) are commonly used in all three tools, but the others are unique for a particular tool. For example, YAGM has three unique biological associations (TF binding association, TF regulation association and mutant phenotype association). STRING has three unique biological associations (gene fusion evidence, cooccurrence, and pathway evidence). GeneMANIA has three unique biological associations (co-inheritance, colocalization, and shared protein domains).

Second, using FKS1 as a query gene, we compare the three lists of top ten associated genes obtained from these three tools when all biological associations are used together. Note that we can only use a single query gene as an example to do the comparison because the

Table 2. Comparison of biological associations constructed in YAGM, STRING and GeneMANIA

\begin{tabular}{|c|c|c|c|}
\hline Biological Association IWeb tool & YAGM & STRING & GeneMANIA \\
\hline Physical Interaction & 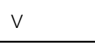 & 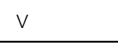 & $v$ \\
\hline Genetic Interaction & $\mathrm{v}$ & $v$ & $\mathrm{v}$ \\
\hline Co-expression & $\mathrm{v}$ & $\mathrm{v}$ & $\mathrm{v}$ \\
\hline Co-citation & $\mathrm{v}$ & $v$ & $v$ \\
\hline TF Binding & $\mathrm{v}$ & & \\
\hline TF Regulation & $v$ & & \\
\hline Mutant Phenotype & $\mathrm{v}$ & & \\
\hline Gene Fusion & & $v$ & \\
\hline Co-occurrence & & $\mathrm{v}$ & \\
\hline Pathway & & $\mathrm{v}$ & \\
\hline Co-inheritance & & & $\mathrm{v}$ \\
\hline Co-localization & & & $v$ \\
\hline Shared Protein Domains & & & $v$ \\
\hline Gene Neighbourhoods & & $\mathrm{v}$ & $\mathrm{v}$ \\
\hline
\end{tabular}

query results of STRING and GeneMANIA cannot be downloaded for many query genes at once. As shown in Figure 5, one gene (GSC2) is predicted as an associated gene of FKS1 by all three tools, but the others are unique for a particular tool. For example, five genes (SLT2, CNA1, CMP2, ROM2 and MNN10) are predicted only by STRING. Five genes (SEC7, PXL1, AIM44, APL1 and $S L X 4)$ are predicted only by GeneMANIA. Nine genes (GAS1, ECM33, SMI1, CCW12, KRE6, PSA1, EXG1, PFK2 and $S C W 4$ ) are predicted only by YAGM. Since our YAGM predicts nine novel associated genes of $F K S 1$, we would like check the biological plausibility of our novel predictions by using the gene description content in SGD database [15]. Seven (GAS1, SMI1, CCW12, KRE6, PSA1, EXG1 and SCW4) of the nine newly predicted associated genes are known to be involved in cell wall process or glucan biosynthesis just like the query gene FKS1, suggesting that YAGM can predict biologically plausible associated genes of a query gene. That is, YAGM can provide alternative candidates of biologically plausible associated genes for biologists to do further experimental investigation.

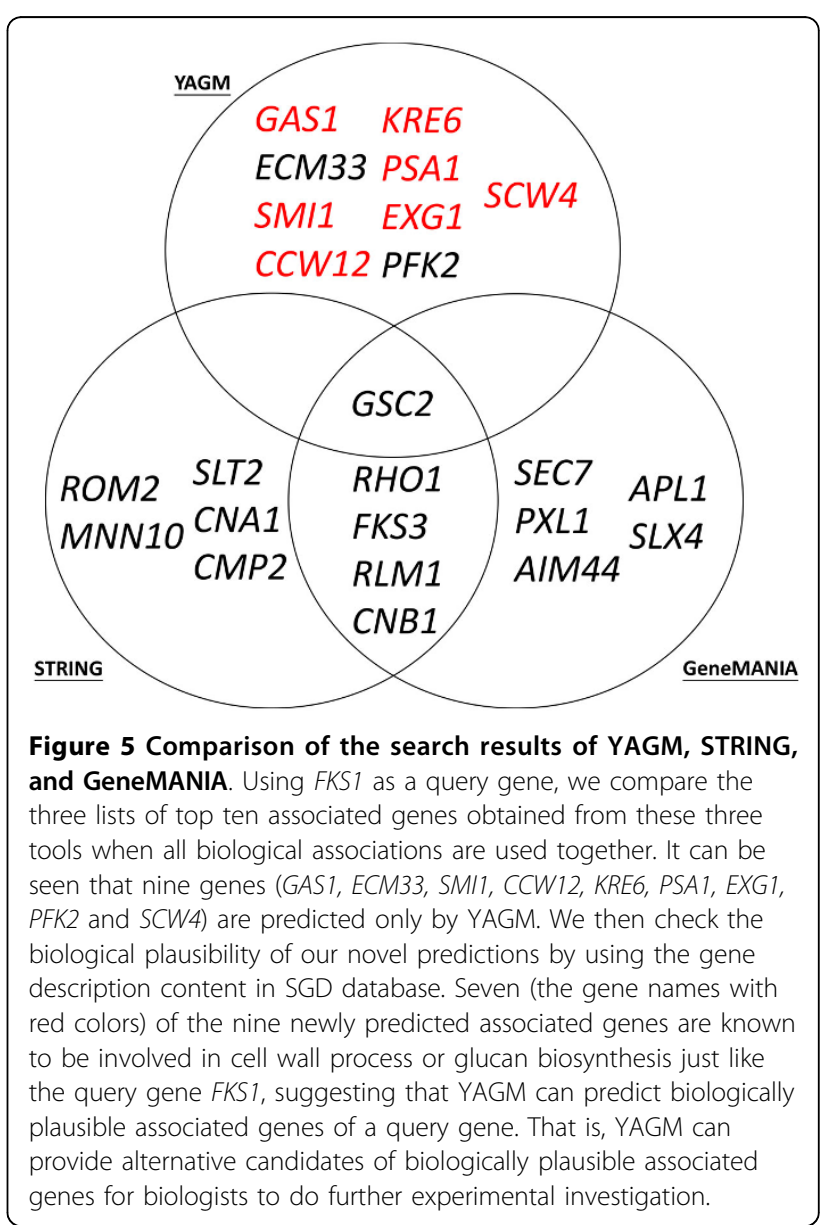




\section{Conclusions}

In this study, we presented the YAGM which provides eight biological associations (including TF binding association, TF regulation association, mutant phenotype association, functional annotation association, physical interaction association, genetic interaction association, and literature evidence association, and expression profile association) for mining associated genes of a query gene in yeast. Among the eight biological associations constructed in YAGM, the first three (TF binding association, TF regulation association, and mutant phenotype association) are novel ones. By comparing the query results of two well-known web tools (STRING and GeneMANIA), we found that YAGM can find out a distinct list of associated genes of a query gene. That is, YAGM can provide alternative candidates of associated genes for biologists to do further experimental investigation. We believe that YAGM will be a useful web tool for yeast biologists. YAGM will be regularly updated based on the newly published literature and the latest release of the YEASTRACT, SGD, BioGRID, and SPELL databases.

\section{Availability and requirements}

YAGM is available at http://cosbi3.ee.ncku.edu.tw/yagm/. The normalized association scores of the eight biological associations between the query gene and every other gene in the yeast genome could be easily downloaded. JavaScript functioning should be enabled in the user-side browsers and the Adobe Flash Player for specific browsers should also be installed. The web interface of YAGM is fully tested on popular browsers: Microsoft IE9, Google Chrome, Apple Safari and Mozilla Firefox. Users are recommended to use these popular browsers for full functionality of YAGM.

\section{Additional material}

Additional file 1: The details of the investigation of the relationships between different biological associations.

\section{Competing interests}

The authors declare that they have no competing interests.

\begin{abstract}
Authors' contributions
WSW conceived the research topic, collected the original datasets, and provided the essential guidance. WSW and CCW wrote the manuscript. CCW constructed the eight biological similarities and the website of YAGM. MJJ and YCW helped to revise the manuscript and the website. All authors have read and approved the final manuscript.
\end{abstract}

\section{Acknowledgements}

This study was supported by National Cheng Kung University and Ministry of Science and Technology of Taiwan MOST-103-2221-E-006 -174 -MY2.

\section{Declarations}

The publication of this paper was funded by National Cheng Kung University and Ministry of Science and Technology of Taiwan MOST-1032221-E-006 -174-MY2.
This article has been published as part of BMC Systems Biology Volume 9 Supplement 6, 2015: Joint 26th Genome Informatics Workshop and 14th International Conference on Bioinformatics: Systems biology. The full contents of the supplement are available online at http://www. biomedcentral.com/bmcsystbiol/supplements/9/S6.

Published: 9 December 2015

\section{References}

1. Clare A, King RD: Predicting gene function in Saccharomyces cerevisiae. Bioinformatics 2003, 19(Suppl 2):ii42-ii49.

2. Hibbs MA, Hess DC, Myers CL, Huttenhower C, Li K, Troyanskaya OG: Exploring the functional landscape of gene expression: directed search of large microarray compendia. Bioinformatics 2007, 23(20):2692-2699.

3. Wu WS, Wei ML, Yeh CM, Chang DT: A regulatory similarity measure using the location information of transcription factor binding sites in Saccharomyces cerevisiae. BMC Syst Biol 2014, 8(Suppl 5):S9.

4. Franceschini A, Szklarczyk D, Frankild S, Kuhn M, Simonovic M, Roth A, et al: STRING v9.1: protein-protein interaction networks, with increased coverage and integration. Nucleic Acids Res 2013, 41(Database issue):D808-D815.

5. Montojo J, Zuberi K, Rodriguez H, Bader GD, Morris Q: GeneMANIA: Fast gene network construction and function prediction for Cytoscape. Flo00Res 2014, 3:153.

6. Chang DTH, Huang CY, Wu CY, Wu WS: YPA: an integrated repository of promoter features in Saccharomyces cerevisiae. Nucleic Acids Res 2011 39(Database issue):D647-D652.

7. Chang DTH, Li WS, Bai YH, Wu WS: YGA: identifying distinct biological features between yeast gene sets. Gene 2012, 518(1):26-34

8. Chiu CC, Chan SY, Wang CC, Wu WS: Missing value imputation for microarray data: a comprehensive comparison study and a web tool. BMC Syst Biol 2013, 7(Suppl 6):S12.

9. Yang TH, Wang CC, Wang YC, Wu WS: YTRP: a repository for yeast transcriptional regulatory pathways. Database 2014, Article ID bau014.

10. Yang TH, Chang HT, Hsiao ESL, Sun JL, Wang CC, Wu HY, Liao PC, Wu WS iPhos: toolkit to streamline the alkaline phosphatase assisted comprehensive LC-MS phosphorproteome investigation. BMC Bioinformatics 2014, 15(Suppl 16):S10.

11. Yang TH, Wang CC, Hung PC, Wu WS: cisMEP: an integrated repository of genomic epigenetic profiles and cis-regulatory modules in Drosophila. BMC Syst Biol 2014, 8(Suppl 4):S8.

12. Hung PC, Yang TH, Liaw HJ, Wu WS: YNA: an integrative gene mining platform for studying chromatin structure and its regulation in Yeast. BMC Genomics 2014, 15(Suppl 9):S5.

13. Lai FJ, Chang HT, Wu WS: PCTFPeval: a web tool for benchmarking newly developed algorithms for predicting cooperative transcription factor pairs in yeast. BMC Bioinformatics 2015.

14. Abdulrehman D, Monteiro PT, Teixeira MC, Mira NP, Lourenço AB, dos Santos SC, et al: YEASTRACT: providing a programmatic access to curated transcriptional regulatory associations in Saccharomyces cerevisiae through a web services interface. Nucleic Acids Res 2011, 39(Database issue):D136-D140.

15. Costanzo MC, Engel SR, Wong ED, Lloyd P, Karra K, Chan ET, et al: Saccharomyces Genome Database provides new regulation data. Nucleic Acids Res 2014, 42(Database issue):D717-D725.

16. Ruepp A, Zollner A, Maier D, Albermann K, Hani J, Mokrejs M, et al: The FunCat, a functional annotation scheme for systematic classification of proteins from whole genomes. Nucleic Acids Res 2004, 32(18):5539-5545.

17. Stark C, Breitkreutz BJ, Chatr-Aryamontri A, Boucher L, Oughtred R, Livstone MS, et al: The BioGRID Interaction Database: 2011 update. Nucleic Acids Res 2011, 39(Database issue):D698-D704.

18. Bass JIF, Diallo A, Nelson J, Soto JM, Myers CL, Walhout AJ: Using networks to measure similarity between genes: association index selection. Nature Methods 2013, 10(12):1169-1176.

19. Lopes CT, Franz M, Kazi F, Donaldson SL, Morris Q, Bader GD: Cytoscape Web: an interactive web-based network browser. Bioinformatics 2010, 26(18):2347-2348.

doi:10.1186/1752-0509-9-S6-S1

Cite this article as: Wu et al:: YAGM: a web tool for mining associated genes in yeast based on diverse biological associations. BMC Systems Biology 2015 9(Suppl 6):S1. 